\title{
TGF- $\beta 1$ induces autophagy and promotes apoptosis in renal tubular epithelial cells
}

\author{
YANFANG XU ${ }^{1}$, SHUYU YANG ${ }^{2}$, JIYI HUANG ${ }^{3}$, \\ SHIWEI RUAN ${ }^{2}$, ZHANG ZHENG ${ }^{2}$ and JIUMAO LIN $^{2}$ \\ ${ }^{1}$ Department of Nephrology, First Affiliated Hospital, Fujian Medical University, Fuzhou 350005; \\ ${ }^{2}$ Academy of Integrative Medicine, Fujian University of Traditional Chinese Medicine, Fuzhou 350108; \\ ${ }^{3}$ Department of Nephrology, First Affiliated Hospital, Xiamen University, Xiamen 361003, P.R. China
}

Received December 11, 2011; Accepted January 24, 2012

DOI: 10.3892/ijmm.2012.911

\begin{abstract}
Transforming growth factor- $\beta 1$ (TGF- $\beta 1$ ) is a multifunctional cytokine that regulates cell growth, differentiation, apoptosis and autophagy in various cell types. It has been shown that TGF- $\beta 1$-driven autophagy represents a novel mechanism of tubular decomposition, leading to renal interstitial fibrosis. However, the exact mechanism by which TGF- $\beta 1$ regulates autophagy is still poorly understood. In the present study, we investigated the effects of exogenous TGF- $\beta 1$ on cultured human renal proximal tubular epithelial cells (HRPTEpiCs). Presence of TGF- $\beta 1$ in the medium induced accumulation of autophagosomes in a time- and dose-dependent manner as seen by monitoring the marker LC 3 by confocal fluorescence microscopy and immunoblotting. In addition, TGF- $\beta 1$ induced upregulation of autophagy-related genes, Atg5, Atg7 and Beclinl. Importantly, increased generation of reactive oxygen species (ROS) and enhanced expression of NADPH oxidases were found to be associated with the TGF- $\beta 1$-induced autophagy. Conversely, treatment with inhibitors of NADPH oxidase markedly reversed the autophagic effects of TGF- $\beta 1$. Apoptotic effects were evaluated by the TUNEL assay, measuring mitochondrial membrane potential and monitoring expression of the pro- and anti-apoptotic genes, Bim and Bcl-2, respectively. Transcriptional silencing of the above three autophagy-related genes in HRPTEpiCs caused attenuation of TGF- $\beta 1$-mediated apoptosis. Similarly, when autophagy was prevented at an early stage by application of 3-methyladenine, the pro-apoptotic effects of TGF- $\beta 1$ were attenuated. These observations suggest that in HRPTEpiCs TGF- $\beta 1$ promotes autophagy through the generation of ROS, which contributes to its proapoptotic effect.
\end{abstract}

Correspondence to: Dr Yanfang Xu, Department of Nephrology, First Affiliated Hospital, Fujian Medical University, Chazhong Road 20, Fuzhou 350005, P.R. China

E-mail: xuyanfang99@hotmail.com

Key words: transforming growth factor- $\beta$, autophagy, apoptosis

\section{Introduction}

Autophagy and apoptosis are two processes, through which injured/aged cells or organelles are eliminated $(1,2)$. Autophagy, or the 'self-eating' function, is characterized by the presence of abundant double-membraned vacuoles called autophagosomes that sequester cytoplasm and cytosolic organelles, such as mitochondria and endoplasmic reticulum. Subsequently, the autophagosome fuses to a lysosome and its contents and inner membrane are degraded and recycled (3). Autophagy is usually regarded as a protective mechanism for cell survival under various conditions including nutrient deprivation and hypoxia (4). However, increasing evidence suggests that in response to excessive stress autophagy may be detrimental and can lead to cell death (5). Excessive autophagic activity may lead to cellular dysfunctions and induce death by destroying a large proportion of the cytosol and organelles, especially the mitochondria and the endoplasmic reticulum (ER) $(6,7)$. The contribution of autophagy to cell death depends on the threshold of the stimuli. It either constitutes a stress adaptation aimed at suppressing apoptosis or conversely provides an alternative pathway to cell death $(2,8)$.

Tubular injury is the major contributor to reduction of renal function. Nephron loss can initiate from the tubular decomposition, followed by glomerular degeneration (9). A growing body of evidence indicates that nephron loss is closely correlated with occurrence of glomeruli, that are atubular or are connected to vestigial tubules in cases of obstructive uropathy and even in primary glomerular diseases $(9,10)$. The process of tubular decomposition starts with tubular shrinkage and atrophy, followed by detachment of the cells from the tubular basement membrane (TBM), finally resulting in almost cellfree, wrinkled profiles of TBM. Li et al showed that in the process of tubular decomposition in obstructive uropathy, epithelial cells undergo autophagy (10). Koesters et al suggested that TGF- $\beta 1$-driven autophagy may represent a novel mechanism of tubular decomposition (11). Thus, in certain renal pathological conditions, increased autophagy of renal tubular epithelial cells may exceed the capability for self protection, such that cell death results from excessive cannibalism, in turn leading to total decomposition of tubular cells and tubular atrophy. 
TGF- $\beta 1$ acts as a multifunctional cytokine that regulates a number of physiological processes including growth, chemotaxis, differentiation, and apoptosis in various types of cells in a dose-dependent and cell-specific manner $(12,13)$. Evidence shows that TGF- $\beta 1$ is able to trigger tubular epithelial cells to transform into mesenchymal cells, in a process called epithelial-to-mesenchymal transition (EMT), eventually leading to fibrosis (14). However, the extent of this impact on nephron loss and interstitial fibrosis development in chronic renal disease remains controversial. Autophagy induced by TGF- $\beta 1$ contributes to excessive cell death, representing a potential mechanism of nephron loss and tubular atrophy. Overexpression of TGF- $\beta 1$ in renal tubules in vivo induces widespread peritubular fibrosis by tubular autophagy and not by the EMT (11). However, whether TGF- $\beta 1$ induces autophagy in renal tubular cells in vitro is not clear. Furthermore, evidence suggests that oxidative stress and generation of reactive oxygen species (ROS) are involved in cellular responses induced by TGF- $\beta 1$, including EMT or mitochondria-dependent apoptosis $(12,14)$. Since oxidative stress is also associated with the induction of autophagy, we would expect a role of ROS in TGF- $\beta 1$-induced autophagy.

Autophagy and apoptosis have both been linked to cell death. Furthermore, apoptosis and autophagy are not mutually exclusive pathways (15). Autophagy may cooperate with the apoptotic machinery or facilitate it, or may even be antagonistic (16). It has been shown that some autophagy related genes (Atg) and Beclinl constitute a point of crosstalk between autophagic and apoptotic pathways. Autophagy has been shown to potentiate the TGF- $\beta 1$-mediated induction of the proapoptotic genes Bim and Bmf in human hepatocellular carcinoma cells and partially contribute to TGF- $\beta 1$-mediated growth inhibition (17). Furthermore, autophagy inhibition by knockdown of Beclin1 or Atg5 or Atg7 significantly suppressed the TGF- $\beta 1$-mediated induction of Bim and Bmf (17). However, the effect of TGF- $\beta 1$ on apoptosis and autophagy in renal tubular cells has not been previously examined. In the present study, we investigated whether TGF- $\beta 1$ could activate autophagy in renal tubular epithelial cells, and examined the role of ROS generation and the convergence of autophagy and apoptosis in this process.

\section{Materials and methods}

Cell culture. All experiments were performed using human renal proximal tubular epithelial cells (HRPTEpiCs) (ScienCell, CA, USA). HRPTEpiCs were maintained in epithelial cell medium (EpiCM, ScienCell Research Laboratories) supplemented with $2 \%$ fetal bovine serum (FBS), at $37^{\circ} \mathrm{C}$ with $5 \% \mathrm{CO}_{2}$.

Materials and reagents. The antibodies used were as follows: anti-LC3-I and anti-LC3-II (Cell Signaling Technology, Danvers, MA); anti-Beclin1 (BD Biosciences); anti-Atg5, anti-Atg7, and $\beta$-actin (Sigma-Aldrich, St. Louis, MO); antiNox4 (Abcam); anti-p22phox, anti-Bax and anti-Bcl-2 (Santa Cruz Biotechnology, CA). The reagents used were as follows: TGF- $\beta 1$ (Sigma-Aldrich) and bafilomycin A1 (BafA1, Santa Cruz Biotechnology); acridine orange, apocynin and diphenyliodonium (DPI) (Sigma-Aldrich). The Trypan Blue Staining
Cell Viability Assay, terminal deoxynucleotidyl transferasemediated dUTP nick translation end labeling (TUNEL) cell apoptosis detection kit, and Hoechst staining kit were provided by the Beyotime Institute of Biotechnology (Haimen, China).

Determination of autophagy by electron microscopy. Autophagy was evaluated by electron microscopy. Cells were harvested gently using buffer containing trypsin and EDTA, washed with PBS, fixed at $4^{\circ} \mathrm{C}$ for $2 \mathrm{~h}$ with $2 \%$ glutaraldehyde, buffered with $0.05 \mathrm{M}$ sodium cacodylate ( $\mathrm{pH} 7.3$ ), embedded in Epon, and prepared for transmission electron microscopy (Hu-12A electron microscope).

Determination of autophagy by LC3. Autophagy was detected using the standard marker, LC3, and staining methods in combination with fluorescence and electron microscopy. The eGFP expression vector, pEGFP-C1 (C1 vector, Invitrogen) was used to clone the amplified LC3 complementary DNAs (cDNAs). LC3 cDNA was amplified by PCR with the following oligonucleotide primers, forward primer: 5'-AATTCTCGAGCCATGCCGTCGGAGAAG AGC-3' and reverse primer: 5'-CCCCGTCGACTTACACT GACAATTTCATCCCG-3', containing restriction enzyme recognition sites that were used in cloning. The HiSpeed Plasmid Maxi kit (Qiagen Inc., Hilden, Germany) was used to purify the plasmids. Transfection was performed with Lipofectamine Plus ${ }^{\mathrm{TM}}$ kit (Invitrogen) according to the manufacturer's recommendation. After transfection, cells were incubated for $16 \mathrm{~h}$ in EpiCM medium, and then treated under various experimental conditions. Images of GFP-LC3 fluorescence were captured under a confocal microscope. The detection of punctated staining with bright fluorescence indicated the presence of GFP-LC3-labeled autophagosomes.

Acridine orange staining of autophagic vacuoles. Acridine orange staining was used to detect the formation of acidic vesicular organelles (AVOs) associated with cell autophagy (5). After treatment with different stimuli, cells were stained with $5 \mu \mathrm{g} / \mathrm{ml}$ acridine orange for $15 \mathrm{~min}$, washed with PBS, and then examined under an inverted fluorescence microscope. Occurrence of autophagy was graded semiquantitatively by counting the number of orange vacuoles and measuring the intensity of orange fluorescence. These measurements were performed by 2 independent observers who were blinded to the experimental conditions. A total of 200 cells were examined at random for each experimental condition.

siRNA synthesis and transfection. Beclinl siRNA, Atg5 siRNA and $A \operatorname{tg} 7$ siRNA (Invitrogen, Carlsbad, CA, USA) were transfected into HRPTEpiCs as previously described (18). RT-PCR and immunoblotting were used to confirm the inhibition of expression of these genes. The oligonucleotides used to generate the Stealth ${ }^{\mathrm{TM}}$ siRNAs for use in this experiment were as follows: Beclin1-565 (named after the position of the translation start site): sense 5'-CCACUCUGUGAGGAAUGCA CAGAUA-3', and antisense 5'-UAUCUGUGCAUUCCUCA CAGAGUGG-3'; Atg5-377: sense 5'-UCGAGAUGUGUGG UUUGGACGAAUU-3', and antisense 5'-AAUUCGUCCAA ACCACACAUCUCGA-3'; Atg7-686: sense 5'-CAGAAGGA GUCACAGCUCUUCCUUA-3' and antisense 5'-UAAGGAA 
A

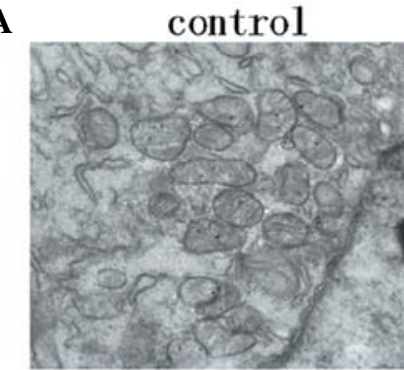

$\mathbf{B}$

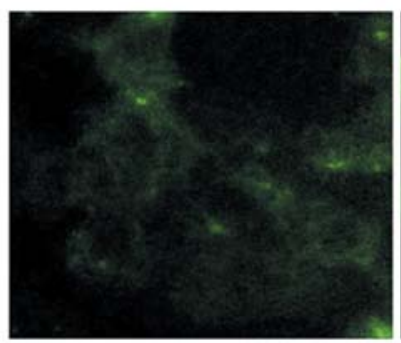

TGF- $\beta 1$

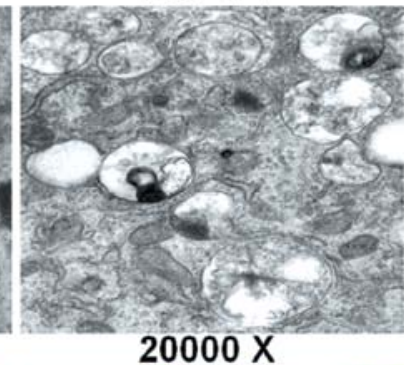

$20000 X$

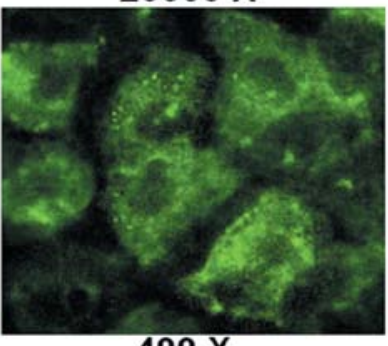

$400 X$

Figure 1. TGF- $\beta 1$ induces autophagy in HRPTEpiCs. HRPTEpiCs were incubated in standard medium (control) or medium containing $10 \mathrm{ng} / \mathrm{ml} \mathrm{TGF}-\beta 1$ for $36 \mathrm{~h}$ and visualized by (A) electron microscopy. (B) HRPTEpiCs expressing the autophagy marker, GFP-LC3, were incubated as above and punctate GFPLC3 fluorescence was observed. Numbers indicate the magnification.

GAGCUGUGACUCCUUCUG-3'. The transfected cells were used for experiments after $24-48 \mathrm{~h}$.

RNA extraction and reverse transcription polymerase chain reaction. Total RNA was extracted from cells using TRIzol reagent (Invitrogen) according to the manufacturer's instructions. First-stand cDNA was synthesized using oligonucleotide primers and M-MLV reverse transcriptase (Promega, Madison, WI, USA) before PCR amplification. Appropriate PCR primer sets for Beclin1, Atg5, Atg7, and $\beta$-actin were synthesized. PCR products were resolved on $1.5 \%$ agarose gels and visualized by ethidium bromide staining, and bands then were analyzed with a Syngene bioimaging system (Bio-Rad, Hercules, CA, USA).

Immunoblot analysis. Cultured cells were washed with icecold PBS and lysed in RIPA lysis buffer (50 mM Tris, pH 7.4, $150 \mathrm{mM} \mathrm{NaCl}, 1 \%$ Triton $\mathrm{X}-100,1 \%$ sodium deoxycholate, and $0.1 \%$ SDS). The protein concentrations were determined by a BCA protein assay kit (Beyotime, Haimen, China). Equal amounts of protein samples were loaded per well, resolved by $10 \%$ SDS-PAGE, and electrophoretically transferred to nitrocellulose membranes (Bio-Rad). The blots were allowed to react with specific antibodies, and the crossreaction was detected by ECL chemiluminescence system (GE Healthcare, Piscataway, NJ, USA). The detailed procedures have been previously described (18).

Measurement of ROS in HRPTEpiCs. The oxidant-sensitive probe, 2',7'-dichlorodihydrofluorescein diacetate (H2DCFDA), was used to detect intracellular reactive oxygen species (ROS). After exposure to the different experimental conditions, cells in 24-well plates were rinsed with PBS, and then incubated with $10 \mu \mathrm{g} / \mathrm{ml} \mathrm{H} 2 \mathrm{DCFDA}$ at $37^{\circ} \mathrm{C}$ for $30 \mathrm{~min}$. Fluorescence intensity was measured using a fluorescence microplate reader.

Fluorescence data were expressed as a ratio of fluorescence intensity in treated samples to that in the control samples.

Measurement of cell viability and apoptosis. Cell viability was measured by the Trypan Blue Staining Cell Viability assay. After treatment with varying doses of TGF- $\beta 1$ for $12-48 \mathrm{~h}$, cells were scraped and resuspended, then incubated with the Trypan blue solution, finally counted in a hemocytometer under a light microscope, and percentage of cell viability was calculated. Apoptotic cells were measured by the TUNEL assay. The 3'-ends of fragmented DNA in apoptotic HRPTEpiCs were labeled using the One Step TUNEL Apoptosis Assay kit. After the experimental treatment, cells were washed with PBS, permeabilized with $0.1 \%$ Triton X-100, and used for a TUNEL assay for $60 \mathrm{~min}$ at $37^{\circ} \mathrm{C}$. Cells were observed under a fluorescence microscope with a 488-nm excitation and a 530-nm emission filter (18).

Apoptosis was also evaluated by staining nuclei with Hoechst stain to measure those with condensed chromatin. HRPTEpiCs were cultured in 6-well flat-bottom plates. After exposure to the different experimental conditions, cells were fixed, washed with PBS, and then stained with Hoechst-33258 $(0.5 \mathrm{ml} /$ well $)$ for $5 \mathrm{~min}$. Cells were observed under a fluorescence microscope with a 350-nm excitation and a 460-nm emission filter.

Measurement of mitochondrial membrane potential. To measure mitochondrial depolarization in HRPTEpiCs, a JC-1 probe was employed. JC-1 exhibits potential-dependent accumulation in mitochondria, which is indicated by a fluorescence emission shift from 529 to $590 \mathrm{~nm}$. Mitochondrial depolarization is indicated by an increase in the ratio of green:red fluorescence intensity, as described previously (18). Cultured cells were rinsed with medium, and then loaded with JC-1. After incubation for $20 \mathrm{~min}$ at $37^{\circ} \mathrm{C}$, cells were examined under a fluorescence microscope.

Statistical analysis. All measurements were based on at least three separate experiments, and values are expressed as mean \pm SD. Data were analyzed by using one-way analysis of variance (ANOVA) followed by a Bonferroni test. Statistically significant level was defined as $\mathrm{P}<0.05$.

\section{Results}

TGF- $\beta 1$ induces autophagy in HRPTEpiCs. To determine whether TGF- $\beta 1$ induces autophagy, HRPTEpiCs were incubated in a medium containing either $10 \mathrm{ng} / \mathrm{ml}$ TGF- $\beta 1$ or its vehicle, for $36 \mathrm{~h}$. Subsequently, cells were visualized by transmission electron microscopy (Fig. 1A). Autophagosomes were recognized as characteristic double-membraned vacuolar structures containing various cytoplasmic elements. Compared with control cells, TGF- $\beta 1$-treated cells displayed a significantly higher number of autophagosomes.

Autophagic activity is marked by the conversion of microtubule associated protein, LC3-I that is localized in the cytosol to LC3-II that is incorporated into the autophagosomal membrane. The amount of LC3-II correlates with the number of autophagosomes and is used to monitor autophagy (5). When HRPTEpiCs expressing GFP-tagged LC3-I were exposed to 
A

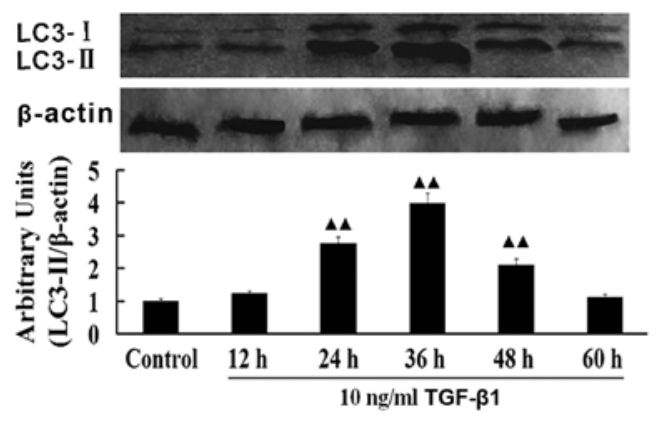

C

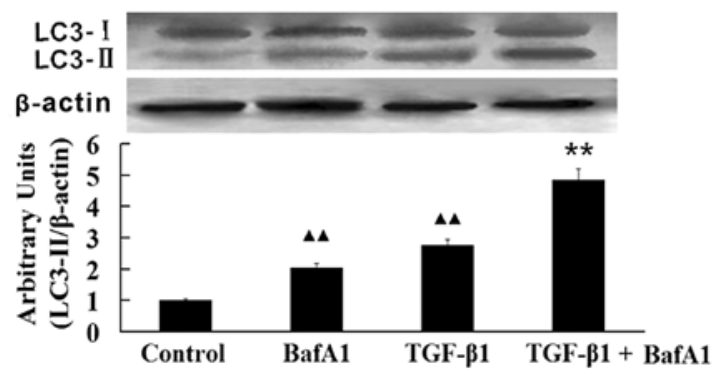

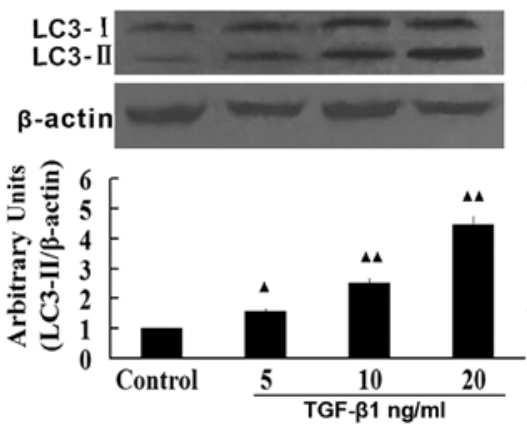

Figure 2. TGF- $\beta 1$ enhances LC3-I to the LC3-II conversion in HRPTEpiCs. HRPTEpiCs were incubated in standard medium (control) or medium containing (A) TGF- $\beta 1,10 \mathrm{ng} / \mathrm{ml}$ for $12-60 \mathrm{~h}$ or (B) TGF- $\beta 1,5-20 \mathrm{ng} / \mathrm{ml}$ for $36 \mathrm{~h}$, to monitor the time- and dose-dependent, responses, respectively. (C) HRPTEpiCs were incubated in standard medium (control) or medium containing TGF- $\beta 1(10 \mathrm{ng} / \mathrm{ml})$ for $36 \mathrm{~h}$ followed by treatment with BafAl (20 nmol/l) for $2 \mathrm{~h}$, as indicated. The contents of endogenous LC3-I and LC3-II were visualized using specific antisera on immunoblots and quantified by densitometry. $\beta$-actin served as a normalizing control. Values plotted are mean $\pm \mathrm{SD}$ of LC3-II/ $\beta$-actin ratios from three independent experiments. ${ }^{\wedge} \mathrm{P}<0.05,{ }^{\wedge} \mathrm{P}<0.01$ vs. the control group; ${ }^{* * *} \mathrm{P}<0.01$ vs. the TGF- $\beta 1$ group.

A

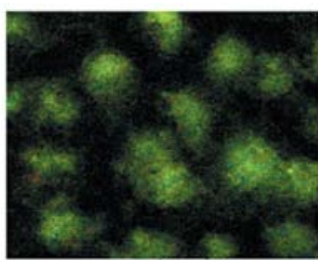

control

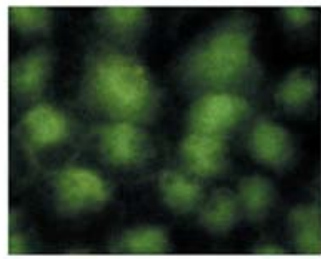

control +3-MA

B

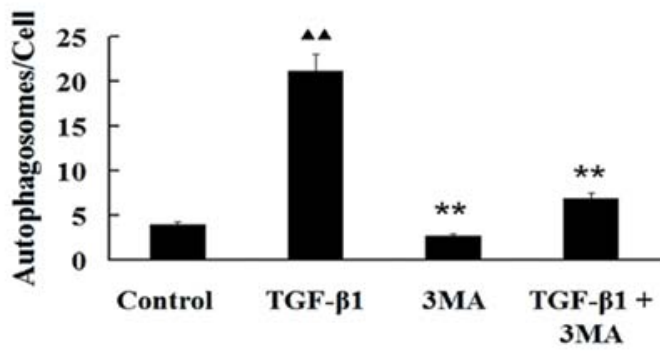

Figure 3. 3-Methyladenine (3-MA) inhibits TGF- $\beta 1$-induced autophagy. HRPTEpiCs were incubated in standard medium (control) or medium containing TGF- $\beta 1(10 \mathrm{ng} / \mathrm{ml}), 3-\mathrm{MA}(10 \mathrm{mM})$, or TGF- $\beta 1+3-\mathrm{MA}$ for $36 \mathrm{~h}$, as indicated. Subsequently, cells were stained with acridine orange to visualize acidic vesicular organelles (AVOs) associated with cell autophagy and observed by fluorescence microscopy. (A) TGF- $\beta 1$ promoted vacuole formation (indicated by orange staining). This effect of TGF- $\beta 1$ was inhibited in the presence of 3-MA. (B) Quantitative analysis of orange fluorescence in the autophagic vacuoles. Values plotted are mean \pm SD from 3 independent experiments. ${ }^{\wedge} \mathrm{P}<0.01$ vs. control group; ${ }^{* *} \mathrm{P}<0.01$ vs. TGF- $\beta 1$ group.
TGF- $\beta 1$ (10 ng/ml for $36 \mathrm{~h}$ ), GFP-LC3-II dots could be seen in autophagosomes (Fig. 1B).

Time- and dose-dependent effects of TGF- $\beta 1$ on autophagy were monitored by measuring the cellular content of both LC3-I and II (Fig. 2). The contents of LC3-II to LC3-I increased steadily up to $36 \mathrm{~h}$, and declined thereafter (Fig. 2A). Similarly, the relative amount of LC3-II increased in a dosedependent manner between $5-20 \mathrm{ng} / \mathrm{ml}$ of TGF- $\beta 1$ (Fig. 2B). Bafilomycin A1 (BafA1), an inhibitor of vacuolar $\mathrm{H}^{+}$-ATPase, blocks the autophagosome-lysosome fusion. The amount of endogenous LC3-II delivered to the lysosomes was seen to increase significantly when HRPTEpiCs were incubated with TGF- $\beta 1$ and BafA1 together, as compared with treatment with either TGF- $\beta 1$ or BafA1 alone (Fig. $2 \mathrm{C}$ ). This synergistic effect on LC3-II conversion of a lysosomal inhibitor supports the hypothesis that autophagic flux is enhanced by TGF- $\beta 1$.

3-Methyladenine inhibits TGF- $\beta 1$-induced autophagy. 3-Methyladenine (3-MA) is an inhibitor of phosphoinositide 3-kinase (PI3K), and is known to inhibit autophagy. HRPTEpiCs were incubated with TGF- $\beta 1$ and/or 3-MA for $36 \mathrm{~h}$ (Fig. 3). TGF- $\beta 1$-treated cells showed a higher number of vacuoles compared with untreated control cells. However, in the presence of 3-MA the number of vacuoles was significantly reduced (Fig. 3). These findings indicate that the proautophagic effect of TGF- $\beta 1$ was inhibited by 3 -MA.

Role of Atg genes in TGF- $\beta$-induced activation of autophagy. HRPTEpiCs were incubated with TGF- $\beta 1(5-20 \mathrm{ng} / \mathrm{ml})$ for $36 \mathrm{~h}$ and expression levels of autophagy-related genes, Beclin1, Atg5, and Atg7 were monitored by RT-PCR and immunoblotting. With increasing concentrations of TGF- $\beta 1$ 
A

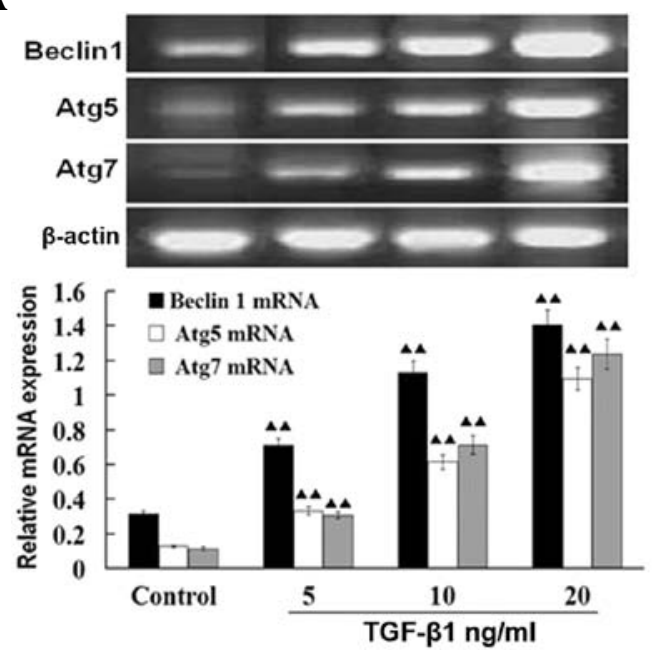

B

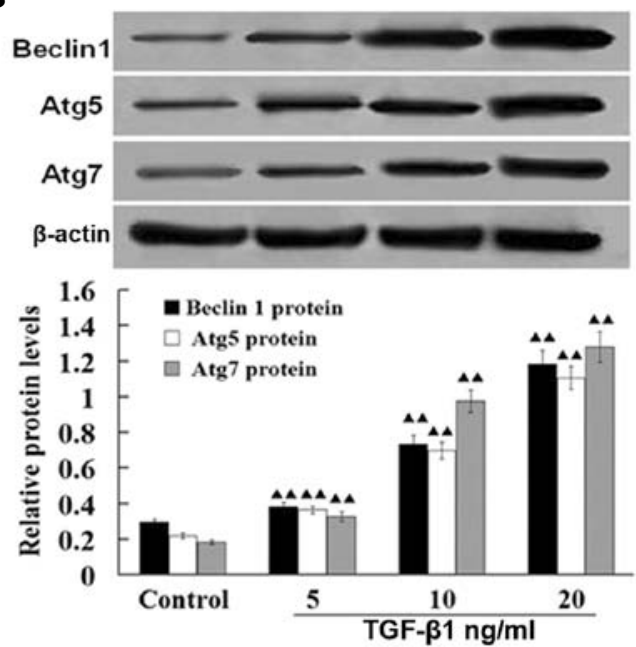

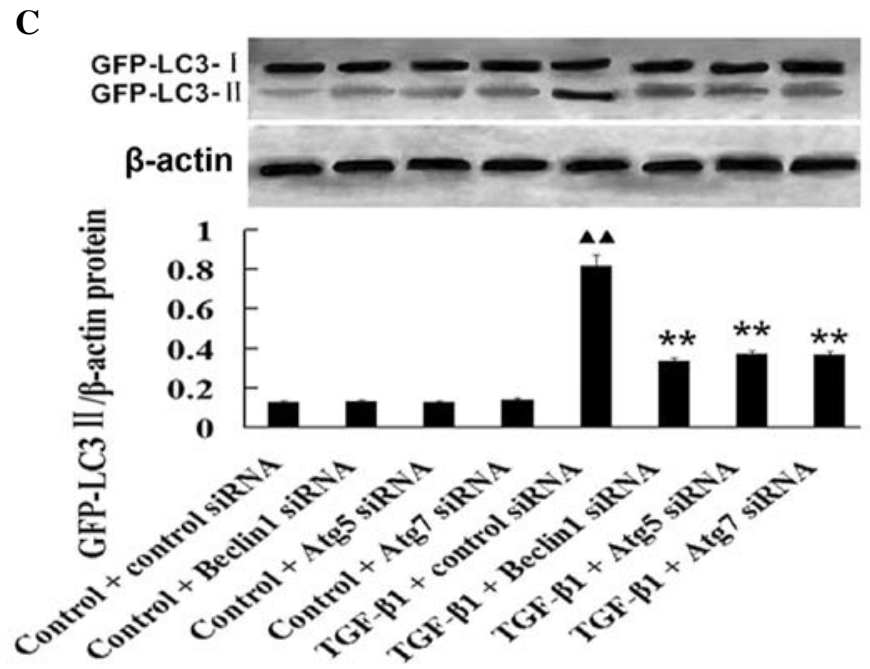

Figure 4. Transcriptional silencing of autophagy-related genes reverses TGF- $\beta 1$-induced autophagy. HRPTEpiCs were incubated in standard medium (control) or medium containing TGF- $\beta 1,5-10 \mathrm{ng} / \mathrm{ml}$ for $36 \mathrm{~h}$ and expression levels of autophagy-related genes, Beclin1, Atg5, and Atg7 were analyzed by (A) RT-PCR analysis. (B) Expression of the respective proteins was analyzed using specific antisera on immunoblots. Transcript and protein levels were normalized with respect to the levels of $\beta$-actin. (C) GFP-LC3-II expressing HRPTEpiCs were transfected with specific siRNAs against Beclin1, Atg5 or Atg7 or a non-specific siRNA (control siRNA), and incubated in standard medium (control) or medium containing TGF- $\beta 1,10 \mathrm{ng} / \mathrm{ml}$ for $36 \mathrm{~h}$, as indicated. The conversion of LC3-I to LC3-II was monitored on immunoblots and quantified as above. ${ }^{\wedge} \mathrm{P}<0.01$ vs. control+control siRNA, control+Beclin1siRNA, control+Atg5 siRNA or control+Atg7 siRNA group; ${ }^{* *} \mathrm{P}<0.01$ vs. TGF- $\beta 1+$ control siRNA group.

in the medium, the transcript and protein levels of all three genes increased in a dose-dependent manner (Fig. 4A and B). Next, HRPTEpiCs were transfected with specific siRNAs of the above genes and the TGF- $\beta 1$-induced conversion of LC3-II was monitored. The content of LC3-II increased after treatment with TGF- $\beta 1$ (10 ng/ml for $36 \mathrm{~h})$ as expected, but was significantly reduced if the expression of Beclin1, Atg5 and Atg7 was suppressed by siRNA (Fig. 4C). Thus, TGF- $\beta 1$ appears to induce autophagy in HRPTEpiCs by induction of the above genes.

Role of oxidative stress in TGF- $\beta 1$-induced autophagy in HRPTEpiCs. Reactive oxygen species (ROS) have been shown to mediate TGF- $\beta 1$-induced diverse biological effects in various cell types (12). In HRPTEpiCs, treatment with TGF- $\beta 1$ at $10 \mathrm{ng} / \mathrm{ml}$ for $10-60 \mathrm{~min}$ resulted in a time-dependent increase in cellular ROS content (Fig. 5A). TGF- $\beta 1$ also increased intracellular ROS generation in a dose-dependent manner (Fig. 5B). NADPH oxidase activation is a major source of ROS in renal cells. Hence, the contents of Nox4 and its subunit p22phox were determined in HRPTEpiCs treated with TGF- $\beta 1$. The content of both the subunits increased in a dose-dependent manner (Fig. 5C). In contrast, when HRPTEpiCs were treated with apocynin or diphenyliodonium (DPI), two NADPH oxidase inhibitors, in the presence of TGF- $\beta 1$ (10 ng/ml for $36 \mathrm{~h}$ ) the expression of autophagy-related genes, Beclin1, Atg5 and Atg7 was attenuated (Fig. 5D). These findings indicate that oxidative stress plays an important role in TGF- $\beta 1$-induced autophagy in renal tubular epithelial cells.

Inhibition of autophagy attenuates TGF- $\beta$-mediated apoptosis. The role of TGF- $\beta 1$-induced autophagy in apoptosis was investigated by different experimental approaches as follows. We first established that HRPTEpiCs treated with different concentrations $(5-20 \mathrm{ng} / \mathrm{ml})$ of TGF- $\beta 1$ for different durations (12-48 h) exhibited a significant decrease in cellular viability 
A

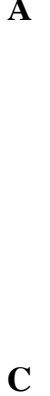

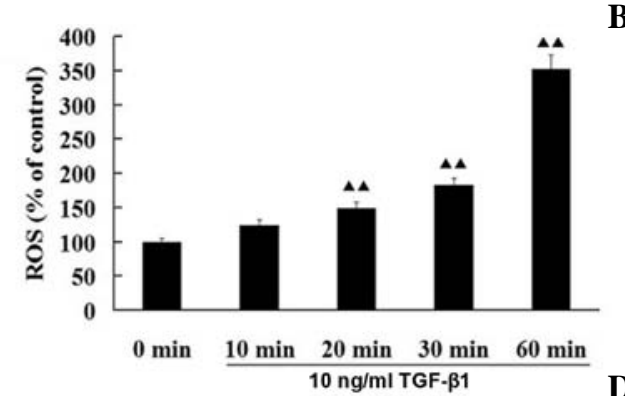

B
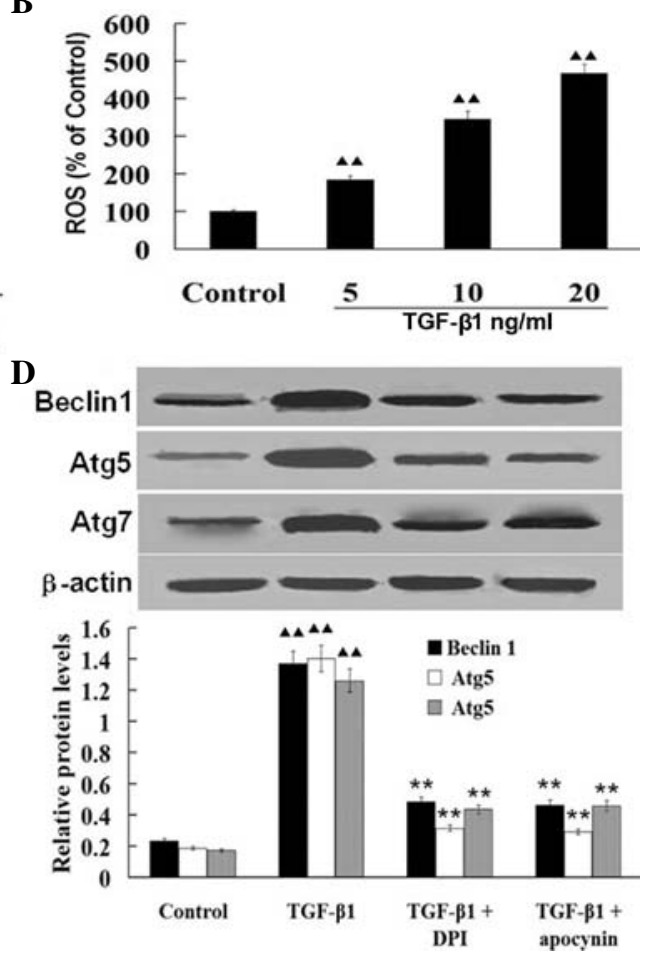

Figure 5. Oxidative stress is associated with TGF- $\beta 1$-induced autophagy. Generation of ROS was monitored in HRPTEpiCs incubated in standard medium (control) or medium containing TGF- $\beta 1,10 \mathrm{ng} / \mathrm{ml}$ for $12-60 \mathrm{~h}$ (A) or TGF- $\beta 1,5-20 \mathrm{ng} / \mathrm{ml}$ for $36 \mathrm{~h} \mathrm{(B)} \mathrm{to} \mathrm{monitor} \mathrm{the} \mathrm{time-} \mathrm{and} \mathrm{dose-dependent,} \mathrm{responses,}$ respectively. ROS generation as measured by the increase in fluorescence of dihydrofluorescein (DCF). (C) HRPTEpiCs were incubated as in (B) and the expression levels of NADPH oxidase subunits, p22phox and NOX4 were determined by immunoblots and quantified as in Fig. 4. (D) HRPTEpiCs were pretreated with NADPH oxidase inhibitors, apocynin $(100 \mu \mathrm{M})$ or diphenyliodonium (DPI, $100 \mathrm{nM})$ for $1 \mathrm{~h}$ before the addition of TGF- $\beta 1(10 \mathrm{ng} / \mathrm{ml}$ for $36 \mathrm{~h})$. The expression levels of the autophagy-related genes were visualized and quantified on immunoblots as in Fig. $4 .{ }^{\wedge}{ }^{\wedge} \mathrm{P}<0.01$ vs. control group; ${ }^{* * *} \mathrm{P}<0.01$ vs. TGF- $\beta 1$ group.

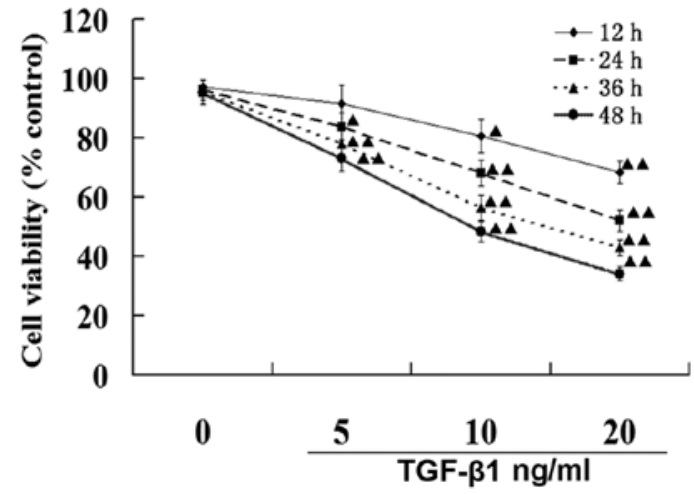

Figure 6. TGF- $\beta 1$ affects cell viability of HRPTEpiCs in a dose- and time-dependent manner. HRPTEpiCs were incubated in standard medium (control) or medium containing TGF- $\beta 1,0-20 \mathrm{ng} / \mathrm{ml}$ for $12-48 \mathrm{~h}$, as indicated. and the number of viable cells were estimated by the Trypan Blue Staining Cell Viability assay. Values plotted are mean \pm SD of triplicate measurements and are representative of three experiments. ${ }^{\wedge} \mathrm{P}<0.05 ;{ }^{\wedge} \mathrm{P}<0.01$ vs. the control group.

in a dose- and time-dependent manner as seen by the trypan blue exclusion assay (Fig. 6). TGF- $\beta 1$-induced autophagy was inhibited either by addition of an inhibitor, 3-MA, or by transcriptional silencing of autophagy-related genes. HRPTEpiCs were incubated in the control medium or medium containing TGF- $\beta 1$ and/or 3-MA for $48 \mathrm{~h}$ and apoptotic cells were visualized by Hoechst staining. Addition of 3-MA reversed the pro-apoptotic effect of TGF- $\beta 1$ (Fig. 7).
HRPTEpiCs transfected with specific siRNAs against Beclinl or Atg5, were treated with $10 \mathrm{ng} / \mathrm{ml}$ TGF- $\beta 1$ for $48 \mathrm{~h}$ and cell viability was determined by the trypan blue exclusion assay. Knockdown of the autophagy-related genes resulted in $\sim 25 \%$ increase in cell viability of TGF- $\beta$-treated HRPTEpiCs (Fig. 8A). The percentage of apoptotic cells was determined by the TUNEL assay in the same cells treated similarly (Fig. 8B and C). TGF- $\beta 1$ induced apoptosis in $\sim 19 \%$ of the cells $(\mathrm{P}<0.01$ compared to control), which was repressed by transcriptional silencing of Beclin1 or Atg5 (Fig. 8B and C). These findings suggest that in HRPTEpiCs the proapoptotic effects of TGF- $\beta 1$ are attenuated by inhibition of autophagy.

Reduction of mitochondrial membrane potential $(\Delta \Psi \mathrm{m})$ and increase in mitochondrial membrane permeability correlate with apoptosis. We measured mitochondrial membrane permeability in HRPTEpiCs transfected with specific siRNAs to knockdown expression of Beclinl or Atg5. Red fluorescence indicates cells with a stable $\Delta \Psi \mathrm{m}$, while green fluorescence indicates the cells with a low $\Delta \Psi \mathrm{m}$. When HRPTEpiCs were exposed to TGF- $\beta 1$, the relative ratio of green fluorescence increased compared with the untreated control cells, indicating that the number of cells undergoing apoptosis increased (Fig. 9A and B). In contrast, in cells in which the autophagy-related genes were silenced, the relative ratio of green fluorescence decreased and that of red fluorescence was restored (Fig. 9A and B). These findings support the notion that inhibition of autophagy reduces the pro-apoptotic effects of TGF- $\beta 1$ in HRPTEpiCs. There is evidence suggesting that the pro-apoptotic molecule, Bim, 
A
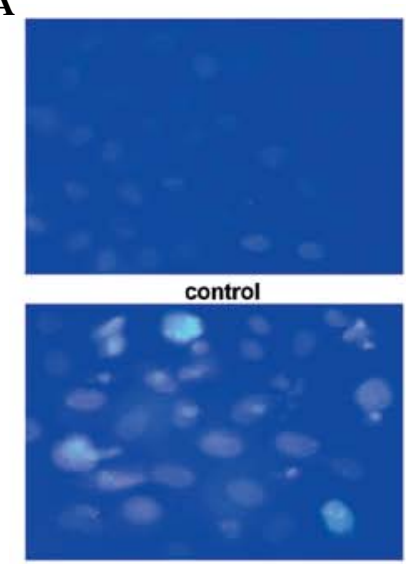

TGF- $\beta 1$

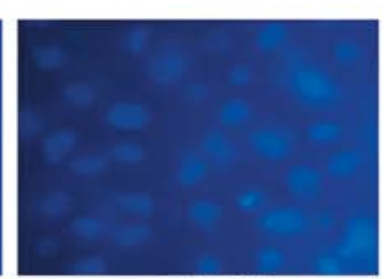

control+3-MA

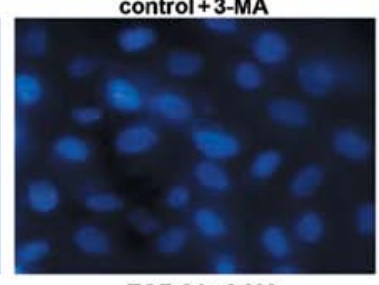

TGF- $\beta 1+3-M A$

B

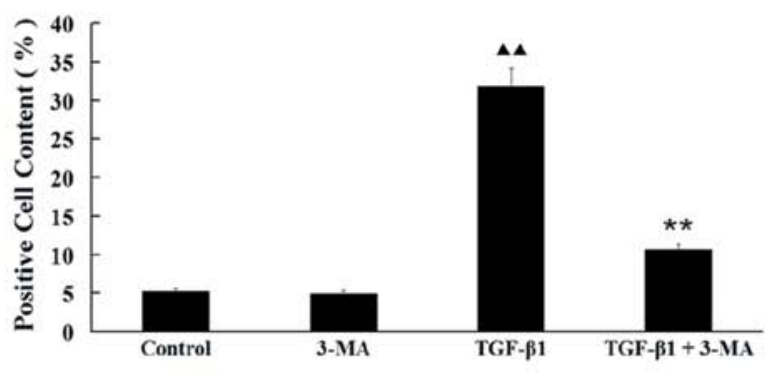

Figure 7. Effect of 3-methyladenine (3-MA) on TGF- 31 -mediated apoptosis in HRPTEpiCs. HRPTEpiCs were incubated in standard medium (control) or medium containing TGF- $\beta 1$ (10 ng/ml), 3-MA (10 mM), or TGF- $\beta 1+3-\mathrm{MA}$ (as indicated) for $48 \mathrm{~h}$. (A) Cells were stained with Hoechst stain to visualize nuclei and (B) the percentage of apoptotic versus viable cells was determined based on chromatin condensation, which indicates cells undergoing apoptosis. ${ }^{\wedge} \mathrm{P}<0.01$ vs. control group; ${ }^{* *} \mathrm{P}<0.01$ vs. TGF- $\beta 1$ group.

A

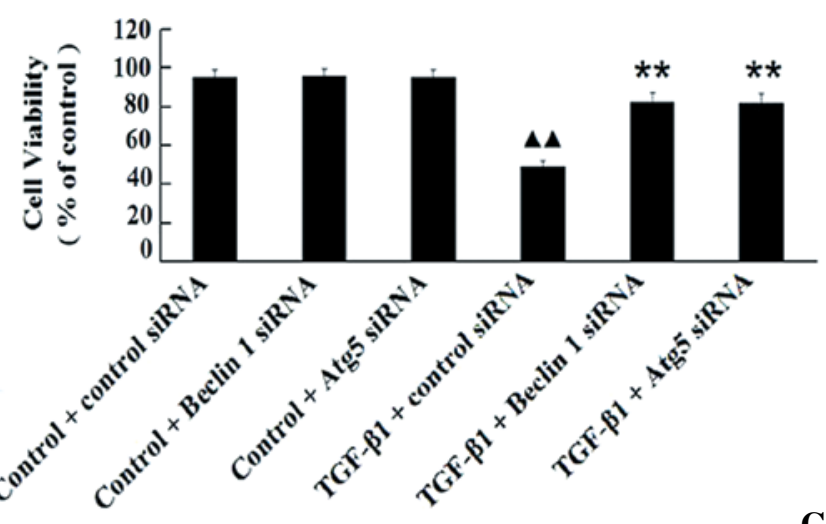

B
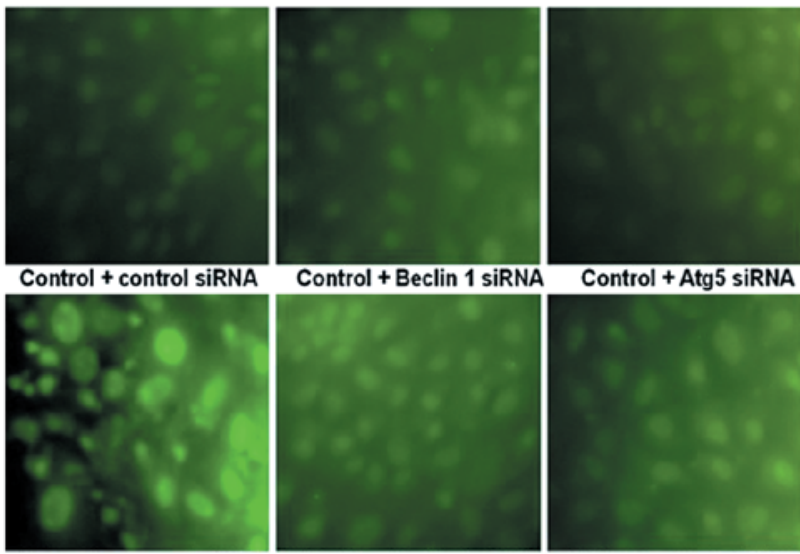

TGF- $\beta 1+$ control siRNA TGF- $\beta 1+$ Beclin 1 siRNA
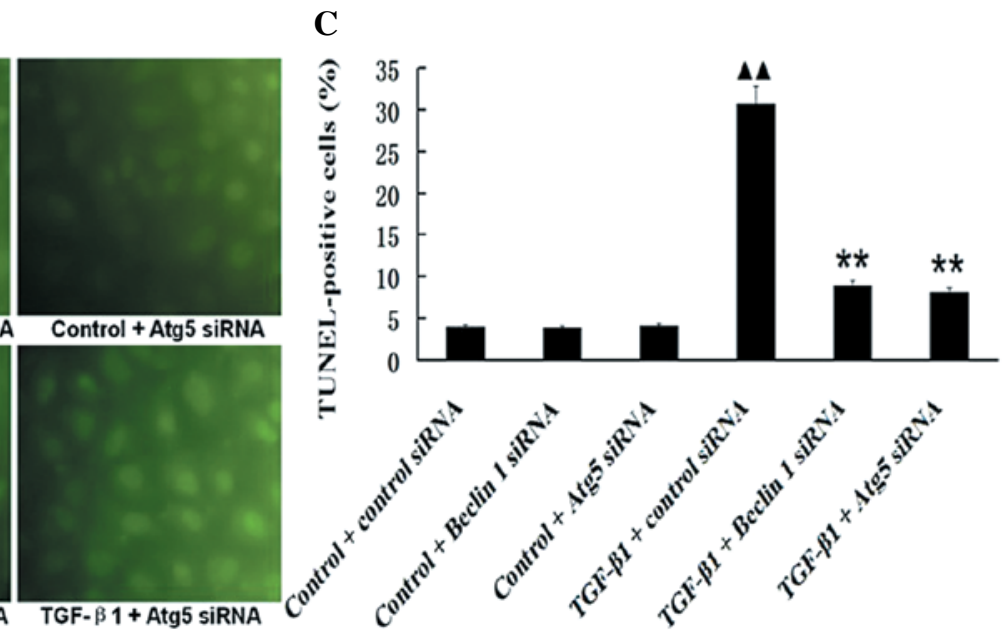

Figure 8. Suppression of autophagy-related genes attenuates TGF- $\beta 1$-mediated apoptosis. HRPTEpiCs were transfected with specific siRNAs against Beclin1, Atg5 or Atg7 or a non-specific siRNA (control siRNA) and incubated in standard medium (control) or medium containing TGF- $\beta 1$ (10 ng/ml) for $48 \mathrm{~h}$. (A) Percentage of viable cells was measured by Trypan Blue Staining Cell Viability assay. Values are means \pm SD of three independent experiments. (B) HRPTEpiCs transfected with specific siRNAs against Beclin1 or Atg5 were treated as above and apoptosis was monitored by TUNEL assay. (C) The percentage of TUNEL-positive cells was calculated relative to the total number of cells. All values are means \pm SD from 10 independent micrographs from each group. ${ }^{\wedge} \mathrm{P}<0.01$ vs. control+control siRNA, control+Beclin1 siRNA, or control+Atg5 siRNA group; ${ }^{* *} \mathrm{P}<0.01 \mathrm{vs.} \mathrm{TGF- \beta 1+} \mathrm{control} \mathrm{siRNA} \mathrm{group.}$

might link autophagy to apoptosis, downstream of TGF- $\beta$ signaling (19). The anti-apoptotic factor, Bcl-2 interacts with Beclin1 and downregulates Beclin1-dependent autophagy (5).
In HRPTEpiCs incubated with TGF- $\beta 1$ there was enhanced expression of the pro-apoptotic gene Bim and reduced expression of the anti-apoptotic gene $\mathrm{Bcl}$-2 (Fig. 9C and D). 
A
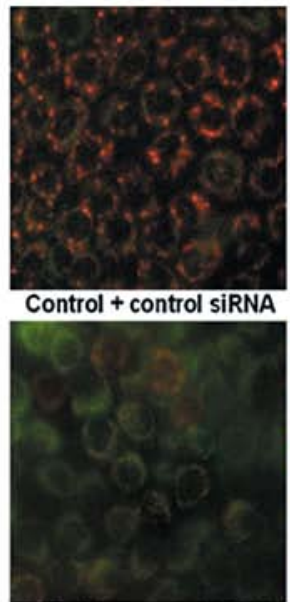

TGF- $\beta 1$ + control siRNA

B
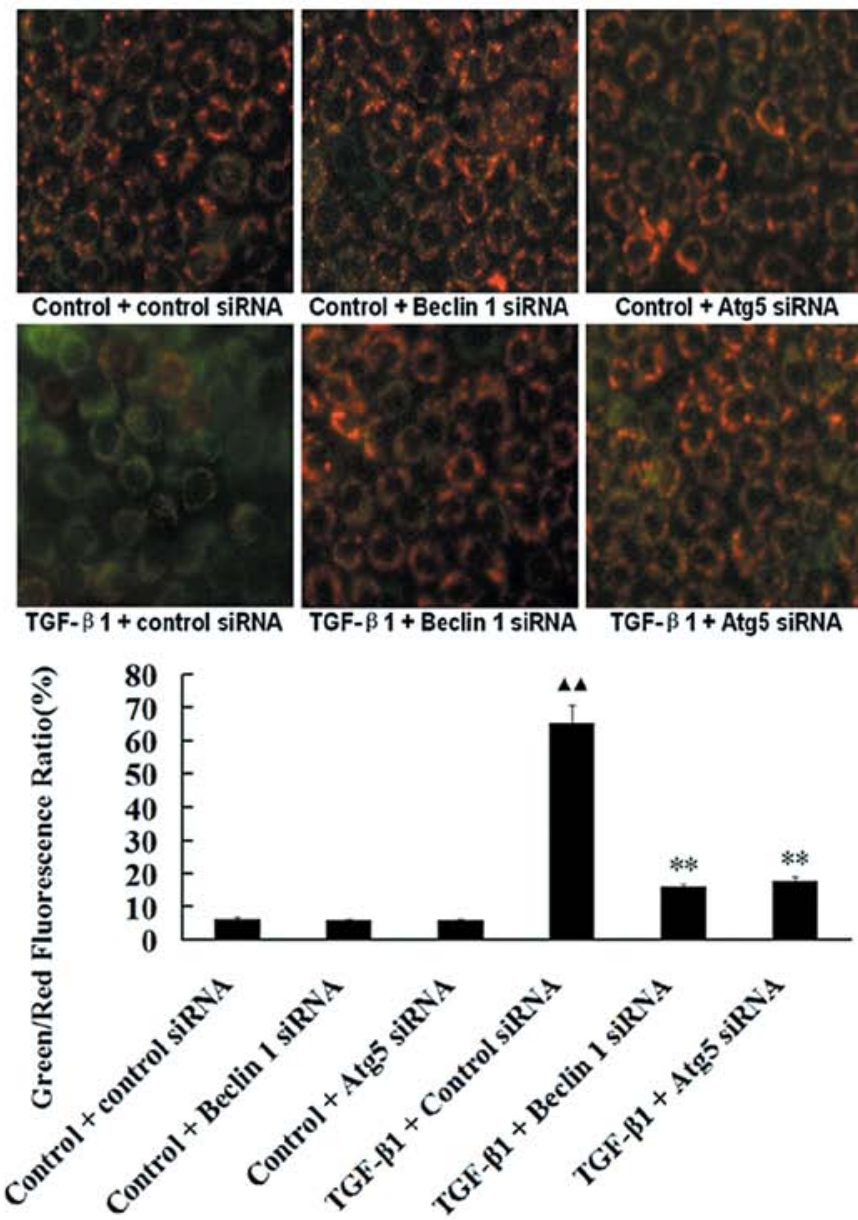

Control + Beclin 1 siRNA
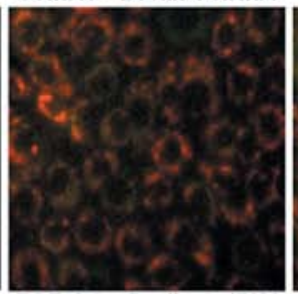

Control + Atg5 siRNA
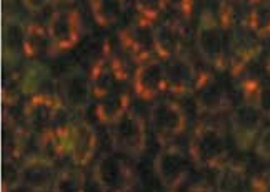

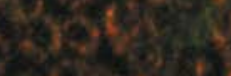

TGF- $\beta 1+$ Atg5 siRNA

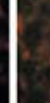

D

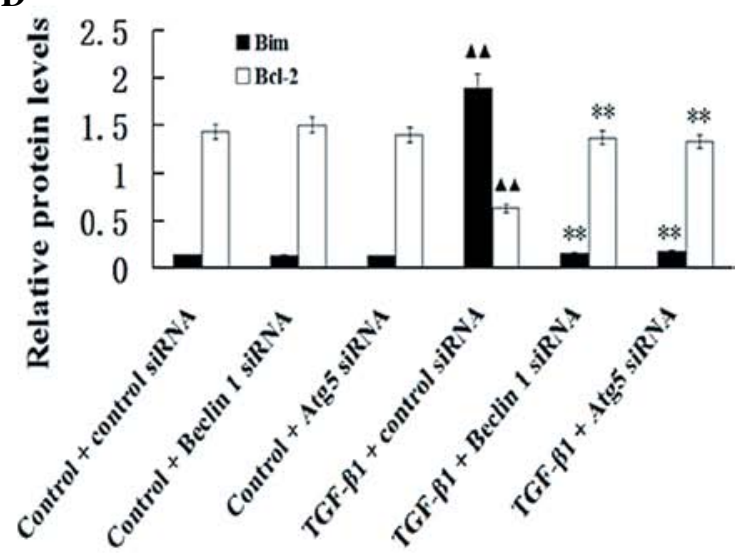

Figure 9. Silencing of autophagy-related genes reverses TGF- $\beta 1$-induced apoptosis in HRPTEpiCs. HRPTEpiCs were transfected with specific siRNAs against Beclinl or Atg5 or a non-specific siRNA (control siRNA) and incubated in standard medium (control) or medium containing TGF- $\beta 1$ (10 ng/ml) for $48 \mathrm{~h}$. (A) Cells were stained with JC-1 and imaged by fluorescence microscopy (magnification, $\mathrm{x} 400$ ). (B) The ratio of green to red fluorescence intensity was quantified as it indicates a reduction in mitochondrial membrane potential, a marker of apoptosis. All values are means \pm SD from 10 independent micrographs from each group. (C) Expression levels of the anti-apoptotic protein, Bcl-2 and the pro-apoptotic protein, Bim were visualized using specific antisera on immunoblots and (D) quantified by densitometry. Protein levels were normalized to those of $\beta$-actin. ${ }^{\wedge} \mathrm{P}<0.01$ vs. control+control siRNA, control+Beclin 1 siRNA, or control+Atg5 siRNA group; ${ }^{* *} \mathrm{P}<0.01$ vs. TGF- $\beta 1+$ control siRNA group.

However, knockdown of autophagy-related genes, Beclinl and Atg5, caused partial suppression of the TGF- $\beta 1$-induced strong expression of Bim and dramatically enhanced the expression of Bcl-2 (Fig. 9C and D).

\section{Discussion}

TGF- $\beta 1$ is a key mediator of response to tubular injury in progressive kidney diseases and regulates both, cell growth and cell death. It has long been appreciated that TGF- $\beta 1$ exhibits diverse functions depending on its dose and cell type, and may have even opposing effects $(13,14,16,20)$. A previous study revealed that overproduction of endogenous TGF- $\beta 1$ in proximal tubular epithelial cells induces widespread peritubular fibrosis and focal nephron degeneration through the autophagy type of programmed cell death (11). Here we investigated the involvement of autophagy induced by TGF- $\beta 1$ in apoptosis in cultured renal epithelial cells. Electron microscopy, the gold standard to monitor the formation of autophagosomes, was used to demonstrate an increased autophagic activity in HRPTEpiCs, with abundant vacuolization, a widely known morphologic indicator for autophagic cell death. The presence of GFP-LC3-labeled autophagosomes and the evaluation of autophagic vacuoles by MDC staining also confirmed this result.

The proautophagic effect of TGF- $\beta 1$ was associated with the expression of LC3-II and transcriptional activation of Atg genes. Formation of autophagosomes includes the formation of preautophagosomal membrane (phagophore or isolation membrane) which is initiated by class III phosphoinositide 3-kinase (PI3K) complex that includes Beclin1, and the elongation of the isolation membrane which is stimulated by two ubiquitin-like conjugation systems (Atg12-Atg5 and LC3-phosphatidylethanolamine) $(3,5)$. Recent studies have indicated that activation of Beclin1 is consistently associated with induction of autophagy in cancer cells (17). Expansion of the membrane starts by conjugation of Atg12 with Atg5 through the action of E1 ubiquitin-activating enzyme, Atg7 and E2 ubiquitin-conjugating enzyme Atg10 (3). Microtubule associated protein light chain 3 (LC3), associated with control elongation of autophagosome, is the second essential ubiquitin-like protein. The cytosolic LC3-I, recruits to the membrane and interacts with phosphatidylethanolamine, 
and is converted to LC3-II. Thus, LC3-II has been used as a marker of autophagy (21). In the present study, autophagy was induced by TGF- $\beta 1$ as directly observed by the accumulation of autophagic vacuoles in the cytoplasm as well as from the increase in the content of the markers, LC3-II, Beclin1, Atg5 and Atg7. That blockade of Atg genes attenuated LC 3 conversion induced by TGF- $\beta 1$ showing that expression of Atg genes is required for TGF- $\beta$-mediated autophagy. We also found that treatment with BafA1, an inhibitor of autophagosomelysosome fusion, together with TGF- $\beta 1$ caused significantly increased accumulation of endogenous LC3-II, indicating that the observed lower level of LC3-I is likely due to its rapid degradation to LC3-II during TGF- $\beta 1$ induced-autophagy.

Reactive oxygen species (ROS) have been shown to mediate TGF- $\beta 1$-induced cellular responses in various cells. Some evidence suggests that TGF- $\beta 1$-induced EMT is mediated by the ROS-MAPK pathway in proximal tubular epithelial cells (14). Interestingly, oxidative stress has also been reported to induce programmed cell death (PCD) including apoptosis or autophagy (3). Some evidence showed that ROS induces autophagy through the class III PI3K/Beclin1 signaling pathways (5). In the present study we showed that TGF- $\beta 1$ induced expression of NADPH oxidase and generation of cellular ROS in a dose- and time dependent manner in HRPTEpiCs. The increase of TGF- $\beta 1$-mediated autophagy-associated gene expression (Beclin1, Atg5 and Atg7) was partly inhibited in the presence of NADPH oxidase inhibitors. Since NADPH oxidase activation is a major source of ROS, these findings suggest that TGF- $\beta 1$-induced autophagy is mediated through ROS generation in renal tubular epithelial cells.

It remains controversial as to whether autophagy is the driver of cell death or a prosurvival process in response to certain stress conditions. Although autophagy was initially described as a cytoprotective mechanism under nutrient deprivation, several lines of evidence have indicated a role for autophagy in promoting cell death $(7,15,22)$, similar to the results presented here in HRPTEpiCs. Some findings have shown that autophagy activation precedes TGF- $\beta$-induced apoptosis while blockade of Atg genes attenuates TGF- $\beta$-mediated apoptosis in hepatoma cells (17). Autophagy plays bidirectional and paradoxical roles in many renal diseases including obstructive uropathy or acute kidney injury $(10,23)$. Hence, we examined the relationship between autophagy and apoptosis in our model. We observed that TGF- $\beta 1$ activates autophagy more potently and continuously in HRPTEpiCs. Hence we speculate that higher and prolonged oxidative stress and/or autophagy induction contributes to TGF- $\beta$-mediated apoptosis in renal tubular epithelial cells.

It has been shown that Atg5 represents a point of crosstalk between autophagic and apoptotic pathways (24). It has been shown that Atg5 promotes autophagy, which is a cytoprotective; however, Atg5 has also been shown to have proapoptotic effects. If overexpressed, Atg5 translocates from the cytosol to the mitochondria, where it associates with the antiapoptotic molecule $\mathrm{Bcl}-\mathrm{xL}$, leading to mitochondrial depolarization and accumulation of ROS and thus triggering apoptosis in tumor cells $(24,25)$. Members of the Beclin1 and Bcl-2 families also serve as a point of crosstalk between the autophagic and apoptotic pathways. Endogenous Beclin1 and Bcl-2 localize primarily in the mitochondria and ER (26). Beclin1 has been identified as a Bcl-2-interacting protein. Beclin1-dependent autophagy is inhibited by overexpression of $\mathrm{Bcl}-2$ and is promoted by silencing of $\mathrm{Bcl}-2$ expression (5). In the present study, we found that siRNA-mediated downregulation of Atg 5 and Beclin1 inhibited apoptosis induced by TGF- $\beta 1$, indicating that in this case autophagy precedes apoptosis. Bim, a member of $\mathrm{Bcl}$-2 family, can induce cell apoptosis by antagonizing the anti-apoptotic effect of Bcl-2, or by interacting with Bax and co-translocating to the mitochondrial membrane, causing an increase in mitochondrial membrane permeability, cytochrome- $\mathrm{C}$ release and further activation of caspases $(17,19)$. Here, in the presence of TGF- $\beta 1$, expression of Bim was induced and that of $\mathrm{Bcl}-2$ was reduced, associated with a loss of mitochondrial membrane potential, all of which agreed with the pro-apoptotic effects of TGF- $\beta 1$. Further, silencing of autophagy-related genes, Atg5 and Beclinl, reversed the TGF- $\beta 1$ modulation of Bim and $B c l-2$ genes, thus linking autophagy and apoptosis via TGF- $\beta 1$. Taken together the data support the notion that autophagy promotes apoptosis under conditions of oxidative stress in HRPTEpiCs.

In conclusion, our results indicate that the pro-apoptotic effects of TGF- $\beta 1$ are probably linked to induction of autophagy and oxidative stress. In cultured human renal proximal epithelial cells, TGF- $\beta 1$ induced the expression of certain autophagy-related genes and stimulated autophagic flux, which was associated with generation of ROS. Induction of autophagy by TGF- $\beta 1$ leading to apoptosis probably explains its effect on tubular decomposition which contributes to fibrosis in chronic renal disease.

\section{Acknowledgements}

This study was supported in part by a Fujian Nature Fund from the Fujian Provincial Department of Science and Technology (no. 2011J01159) and in part by an innovation research fund (no. 2011-CX-10) from the Department of Health of Fujian Province of China.

\section{References}

1. Galluzzi L, Morselli E, Vicencio JM, et al: Life, death and burial: multifaceted impact of autophagy. Biochem Soc Trans 36 : 786-790, 2008

2. Ding WX, Ni HM, Gao W, et al: Differential effects of endoplasmic reticulum stress-induced autophagy on cell survival. J Biol Chem 282: 4702-4710, 2007.

3. Martinet W and De Meyer GRY: Autophagy in atherosclerosis: a cell survival and death phenomenon with therapeutic potential. Circ Res 104: 304-317, 2009.

4. Levine B and Klionsky DJ: Development by self-digestion: molecular mechanisms and biological functions of autophagy. Dev Cell 6: 463-477, 2004.

5. Zhang H, Kong X, Kang J, Su J, Li Y, Zhong JT and Sun LK: Oxidative stress induces parallel autophagy and mitochondria dysfunction in human glioma U251 cells. Toxicol Sci 110: 376-388, 2009.

6. Debnath J, Baehrecke EH and Kroemer G: Does autophagy contribute to cell death? Autophagy 1: 66-74, 2005.

7. Law BY, Wang M, Ma DL, et al: Alisol B, a novel inhibitor of the sarcoplasmic/endoplasmic reticulum $\mathrm{Ca}^{2+}$ ATPase pump, induces autophagy, endoplasmic reticulum stress, and apoptosis. Mol Cancer Ther 9: 718-730, 2010.

8. Gozuacik D and Kimchi A: Autophagy as a cell death and tumor suppressor mechanism. Oncogene 23: 2891-2906, 2004.

9. Javaid B, Olson JL and Meyer TW: Glomerular injury and tubular loss in adriamycin nephrosis. J Am Soc Nephrol 12: 1391-1400, 2001. 
10. Li L, Zepeda-Orozco D, Black R and Lin FM: Autophagy is a component of epithelial cell fate in obstructive uropathy. Am J Pathol 176: 1767-1778, 2010.

11. Koesters R, Kaissling B, LeHir M, et al: Tubular overexpression of transforming growth factor- $\beta 1$ induces autophagy and fibrosis but not mesenchymal transition of renal epithelial cells. Am J Pathol 177: 632-643, 2010.

12. Herrera B, Alvarez AM, Sanchez A, et al: Reactive oxygen species (ROS) mediates the mitochondrial-dependent apoptosis induced by transforming growth factor (beta) in fetal hepatocytes. FASEB J 15: 741-751, 2001.

13. Wang $S$ and Hirschberg R: BMP7 antagonizes TGF- $\beta$-dependent fibrogenesis in mesangial cells. Am J Physiol Renal Physiol 284 F1006-F1013, 2003.

14. Rhyu D, Yang Y, Ha H, et al: Role of reactive oxygen species in TGF- $\beta 1$-induced mitogen-activated protein kinase activation and epithelial-mesenchymal transition in renal tubular epithelial cells. J Am Soc Nephrol 16: 667-675, 2005.

15. Sansanwal P, Yen B, Gahl WA, Ma YW, Ying LH, Wong LJ and Sarwal MM: Mitochondrial autophagy promotes cellular injury in nephropathic cystinosis. J Am Soc Nephrol 21: 272-283, 2010.

16. Eisenberg-Lerner A, Bialik S, Simon HU, et al: Life and death partners: apoptosis, autophagy and the cross-talk between them Cell Death Differ 16: 966-975, 2009.

17. Kiyono K, Suzuki HI, Matsuyama H, et al: Autophagy is activated by TGF-beta and potentiates TGF- $\beta$-mediated growth inhibition in human hepatocellular carcinoma cells. Cancer Res 69: 8844-8852, 2009 .
18. Xu Y, Ruan S, Xie H and Lin J: Role of LOX-1 in Ang II-induced oxidative functional damage in renal tubular epithelial cells. Int J Mol Med 26: 679-690, 2010.

19. Altman BJ, Wofford JA, Zhao Y, et al: Autophagy provides nutrients but can lead to Chop-dependent induction of Bim to sensitize growth factor-deprived cells to apoptosis. Mol Biol Cell 20: 1180-1191, 2009.

20. Ding Y, Kim JK, Kim SI, Na HJ, Jun SY, Lee SJ and Choi ME: TGF- $\beta 1$ protects against mesangial cell apoptosis via induction of autophagy. J Biol Chem 285: 37909-37919, 2010.

21. Kawakami T, Inagi R, Takano H, et al: Endoplasmic reticulum stress induces autophagy in renal proximal tubular cells. Nephrol Dial Transplant 24: 2665-2672, 2009.

22. Gajewska M, Gajkowska B and Motyl T: Apoptosis and autophagy induced by TGF- $\beta 1$ in bovine mammary epithelial BME-UV1 cells. J Physiol Pharmacol 56: 143-157, 2005.

23. Yang C, Kaushal V, Shah SV and Kaushal GP: Autophagy is associated with apoptosis in cisplatin injury to renal tubular epithelial cells. Am J Physiol Renal Physiol 294: F777-F787, 2008.

24. Nishida K, Yamaguchi O and Otsu K: Crosstalk between autophagy and apoptosis in heart disease. Circ Res 103: 343-351, 2008.

25. Yousefi S, Perozzo R, Schmid I, et al: Calpain-mediated cleavage of Atg5 switches autophagy to apoptosis. Nat Cell Biol 8: 1124-1132, 2006.

26. Pattingre S, Tassa A, Qu X, et al: Bcl-2 antiapoptotic proteins inhibit Beclin 1-dependent autophagy. Cell 122: 927-939, 2005. 